\title{
Estimating bacteria diversity in different organs of nine species of mosquito by next generation sequencing
}

\author{
M V Mancini ${ }^{1,3+}$, C Damiani $^{1 \dagger}$, A Accoti ${ }^{2}$, M Tallarita ${ }^{2}$, E Nunzi ${ }^{2}$, A Cappelli ${ }^{1}$, J Bozic ${ }^{1}$, R Catanzani ${ }^{2}$, P Rossi ${ }^{1}$, \\ M Valzano ${ }^{1}$, A Serrao ${ }^{1}$, I Ricci ${ }^{1}$, R Spaccapelo ${ }^{2 *}$ and G Favia ${ }^{1 *}$ (ID
}

\begin{abstract}
Background: Symbiosis in insects is accumulating significant amount of studies: the description of a wide array of mutualistic associations across the evolutionary history of insects suggests that resident microbiota acts as a driving force by affecting several aspects of hosts biology.

Among arthropods, mosquito midgut microbiota has been largely investigated, providing crucial insights on the role and implications of host-symbiont relationships. However, limited amount of studies addressed their efforts on the investigation of microbiota colonizing salivary glands and reproductive tracts, crucial organs for pathogen invasion and vertical transmission of symbiotic microorganisms. Using $16 \mathrm{~S}$ rRNA gene sequencing-based approach, we analysed the microbiota of gut, salivary glands and reproductive tracts of several mosquito species, representing some of the main vectors of diseases, aiming at describing the dynamics of bacterial communities within the individual.
\end{abstract}

Results: We identified a shared core microbiota between different mosquito species, although interesting inter- and intra-species differences were detected. Additionally, our results showed deep divergences between genera, underlining microbiota specificity and adaptation to their host.

Conclusions: The comprehensive landscape of the bacterial microbiota components may ultimately provide crucial insights and novel targets for possible application of symbionts in innovative strategies for the control of vector borne diseases, globally named Symbiotic Control (SC), and suggesting that the holobiont of different mosquito species may significantly vary. Moreover, mosquito species are characterized by distinctive microbiota in different organs, likely reflecting different functions and/or adaptation processes.

Keywords: Symbionts, Mosquitoes, Metagenomics

\section{Background}

Insects represent the most diverse and abundant clade of metazoans, accounting for more than $90 \%$ species and dominating a variety of terrestrial habitats. Diverse insect habits are founded on associations with microorganisms, whose diversity reflects the variety of their hosts [1]. These complex associations are passed between generations and can assume mutualism or commensalism features. The

\footnotetext{
* Correspondence: roberta.spaccapelo@unipg.it; guido.favia@unicam.it ${ }^{\dagger} \mathrm{M} V$ Mancini and C Damiani contributed equally to this work. ${ }^{2}$ Department of Experimental Medicine, Functional Genomics Center, University of Perugia, Via Lucio Severi 1, 06132 Perugia, Italy

${ }^{1}$ School of Biosciences and Medical Veterinary, University of Camerino, Via Gentile III da Varano, 62032 Camerino, MC, Italy

Full list of author information is available at the end of the article
}

most important distinction is whether this microbiota is transient (acquired from the surrounding environment) or it is indigenous to its host, maintaining stable communities and colonizing the gut habitat [2].

Various insects host resident microorganisms able to influence many insect's key functions, although not primarily. Diverse physiological, metabolic and immune processes of hosts are significantly influenced by their microbiota, whose colonization significantly contributed to their evolutionary success. Coexistence and coevolution may rely on increased microbial production of nutrients valuable to the host and correlated changes in its metabolism, promoting host complementarity to microbial nutrients [3]. An elegant study describes symbiotic

(c) The Author(s). 2018 Open Access This article is distributed under the terms of the Creative Commons Attribution 4.0 International License (http://creativecommons.org/licenses/by/4.0/), which permits unrestricted use, distribution, and reproduction in any medium, provided you give appropriate credit to the original author(s) and the source, provide a link to the Creative Commons license, and indicate if changes were made. The Creative Commons Public Domain Dedication waiver (http://creativecommons.org/publicdomain/zero/1.0/) applies to the data made available in this article, unless otherwise stated. 
bacteria of Drosophila melanogaster capable of affecting mating preferences by changing the levels of cuticular hydrocarbons and sex pheromones [4]. Additionally, endosymbionts were found to influence dispersal behaviour, species distribution and overall community composition in spiders [5, 6]. Symbiotic bacteria also impact on body colours of aphids, influencing prey-predator interactions, as well as interactions with other endogenous endosymbionts. Interestingly, insect life cycles are also dependent on microbiome: some mosquito species were reported to rely upon their gut microbiota for molting and developing from larvae to adults [7]. In addition, microbiota contribution on the well-being of the host is also revealed by its ability to promote the resistance of insects to certain natural enemies, including viruses, bacteria, fungi, nematodes, parasites, and in turn, to modulate vector capacity of some insects involved in transmission of infectious diseases. Moreover, symbionts are shown to contribute to insecticide resistance phenomena [8].

In this frame, among insects, mosquitoes represent an important public health challenge in many parts of the globe. Anopheles mosquitoes are vectors of human pathogens, including parasites responsible of severe infectious diseases (malaria and lymphatic filariasis) and arboviruses (O'nyong-nyong virus). In sub-Saharan Africa, the main human malaria vectors are members of the An. gambiae complex, together with An. coluzzii. An. stephensi, instead, represents one of the major malaria vector in Asia.

Mosquitoes of the genus Aedes are worldwide involved in outbreaks of arboviruses, including Dengue, Chikungunya and Zika viruses [9]. Aedes mosquitoes are known to be highly invasive with a great capacity to adapt to contrasting climates and environments. Mosquitoes belonging to the Culex genus were found to be involved in the transmission of human and animal diseases, such as West Nile fever, often acting as a bridge vector between birds and humans [10]. Additionally, these genera exhibit different and specific rhythmic behaviours across their life cycle. For instance, the majority of Anopheles and Culex species are mainly characterized by nocturnal biting behaviour, whereas Aedes typically engage biting activities in the daytime. They also differ in the choice of the breeding site: Anopheles mostly prefer clear water exposed to sunlight, Culex and Aedes are instead mostly found in dark water containing organic matter [11].

Previous studies have described midgut microbiota of mosquitoes as a driving force to directly and/or indirectly affects host-pathogen interactions, and ultimately vector capacity, significantly influencing diseases transmission. In Anopheles mosquitoes, resident microbiota inhibits the invasion of ookinetes of the malaria parasite Plasmodium of the midgut epithelium, reducing infection rate: this effect is mediated by colonizing bacteria, and not caused by their direct interaction with Plasmodium [12]. Also, arboviruses transmission undergoes microbiota-mediated modulations within the mosquito, mainly due to its indirect influence on nutrient catabolism, development, and immune responses [7, 13, 14]. This is particularly remarkable with viruses because of their strict dependence on host factors for invasion and replication processes. Furthermore, microbiota can directly interact with arboviruses by inhibiting viral transmission through the secretion of anti-viral compounds [15]. On the other hand, bacteria could also act as pro-factors by increasing vector permissibility to arboviral infections [16, 17].

Microbiota in mosquitoes is often restricted to bacteria associated to the gut. This tissue represents a reservoir of a wide variety of microbial communities, whose characterization is essential and preparatory for a solid understanding of the overall vector biology [18]. Nevertheless, other organs result to be inhabited by microorganisms: some taxa are shared with the intestinal tract, while some others showed preferences for different tissues. Only recently, a comprehensive study addressed the question about the various and diverse composition of the microbiota of midguts, ovaries, and salivary glands of female individuals of An. gambiae and An. coluzzii, using pyrosequencing analysis of the $16 \mathrm{~S}$ rRNA gene [19]. Additionally, the analysis of the salivary glands of An. culicifacies revealed to host a more complex and various microbiome than that inhabiting the gut [20], underlying diverse bacterial colonization abilities, and in particular the intrinsic environmental divergence offered by organs, despite belonging to the same individual.

We investigated nine species of mosquitoes belonging to the main representative mosquito genera, in terms of geographical diffusion and public health interest. This includes six anopheline species [An. gambiae (G3 and Kisumu strains), An. coluzzii, An. arabiensis, An. quadriannulatus, An. merus, An. stephensi], two belonging to the Aedes genus (Ae. aegypti, Ae. albopictus) and Cx. quinquefasciatus. Herein, data of an extensive study aimed to characterize bacterial microbiota associated to gut, salivary glands and reproductive organs are reported. The importance of describing the microbiota of these organs lies in possibility to develop innovative paratransgenic approaches for vector control. The selection of suitable symbiont candidates primarily requires a specific tissue tropism in the gut, the salivary glands and the reproductive organs [21], since i) the transmission cycle of many pathogens starts in the intestinal tract and culminates with the invasion of the salivary glands from where they are transmitted to a new host and ii) the colonization of the reproductive organs allows the vertical transmission of bacteria to the offspring, ensuring their persistence within a population. 


\section{Results}

\section{Overall distribution of bacteria within different organs}

A total of 951.930 reads were generated after removal of short reads $(<250 \mathrm{bp})$, chimeras and the discard of spurious OTUs [22] from all tissues analyzed. The number of reads varied among samples (minimum $=2228$, maximum $=62,851$ ) (Table 1$)$, therefore the number of sequences for each sample was rarefied to the minimal readings of 2228. The sequences were clustered in 924 bacterial OTUs (Table 1). Analysis of the rarefaction curves indicated an adequate sampling quality; only in a few cases, the curves did not reach the saturation to an asymptote, indicating the presence of additional rare bacteria taxa or further spurious OTUs (Additional file 1: Figure S1). Furthermore, the number of observed species estimator was used to evaluate bacteria richness between the mosquito species/strains (Additional file 2: Figure S2). Interestingly, irregular diversity in terms of bacterial abundance is highlighted when indices are compared. Bacterial richness of reproductive organs is higher than that found in the other tissues (Table 1). In most samples, salivary glands harbour a more diverse microbiota than the gut. In particular, Ae aegypti harbours the highest number of bacteria species compared to others, followed by An. coluzzii and An. quadriannulatus. An. gambiae G3 and An. stephensi contain the lowest number of bacteria species, explained by the long adaptation in laboratory conditions (Additional file 2: Figure S2). In total, mosquito tissues (guts, reproductive organs and salivary glands) host a flora consisting of bacterial OTUs belonging to 12 phyla, 74 families and 121 genera. Among these, 4 phyla represent more than $99 \%$ of the total microbiota: Proteobacteria, Bacteroidetes, Firmicutes and Actinobacteria (Fig. 1, Additional file 3: Table S1). The OTUs assigned to other not abundant phyla $(<1 \%)$ included: Fusobacteria, Acidobacteria, Cyanobacteria, Chlamydiae, Deinococcus-Thermus, Elusimicrobia, Planctomycetes and SHA-109. Some bacteria could not be assigned to any taxa $(0.1 \%)$. The phylum Proteobacteria is dominant in the majority of tissues, showing to be the main constituent of a shared and conserved microbiota core (Fig. 1). Striking examples of Proteobacteria abundance $(>60 \%)$ are found in the following organs: i) guts of females; ii) guts of males, except for An. gambiae G3 and An. coluzzii; iii) salivary glands of all species apart from $A n$. gambiae G3 and $A n$. coluzzii; iv) the reproductive organs of both males and females of An. arabiensis, An. merus, An. stephensi, Ae. albopictus and Ae. aegypti, the ovaries of An. gambiae G3 and organs of $C x$. quinquefasciatus males. However, Bacteroidetes (> 68\%) are present in male guts and salivary glands of both An. gambiae G3 and An. coluzzii, and in the reproductive organs of $A n$. gambiae G3 males. The guts of $A n$. gambiae Kisumu males harbour more than $28 \%$ of Bacteroidetes, which also encompasses $10 \%$ of taxa in Ae. aegypti organs. Firmicutes and Actinobacteria strongly colonize the reproductive organs of both males and females of An. coluzzii, An. quadriannulatus and An. gambiae Kisumu, whereas they are found only in ovaries of Cx. quinquefasciatus.

Consequently, a principal coordinate analysis (PCoA) plot was generated, revealing that the reproductive organs of An. stephensi males and An. gambiae G3 females are divergent from other samples (Fig. 2a). This sharp difference is mostly caused by abundant presence of Serratia. Moreover, the reproductive organs of An. gambiae G3 males show a unique cluster due to the large presence of Elizabethkingia. The relevant presence of members of the Elizabethkingia genus also defines a cluster in the salivary glands of An. gambiae G3 and An. coluzzii (Fig. 2 b); an additional group is composed by guts of An. gambiae G3 males and An. coluzzii females (Fig. 2c). The rest of the samples tends to cluster together (Fig. 2a-b-c ). A global representation is described in the merged picture (Fig. $2 \mathrm{~d}$ ).

\section{Microbiota composition of reproductive organs}

When classified by classes, although irregularly abundant, Alphaproteobacteria are present in most samples, almost representing the total microbiota of Ae. albopic-

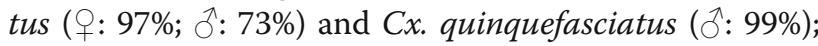
whereas in An. gambiae G3, An. coluzzii males, An. gambiae Kisumu females, it accounts for less than $1 \%$ of the OTUs. Lower abundance is detected in An. arabiensis ( $($ : 33\%, ô: 42\%), An. stephensi ( $9: 48 \%)$, Ae. aegypti ( $q$ and $\delta 32 \%$ ), Cx. quinquefasciatus ( $q$ : 13\%) and between 1 and $6 \%$ in the remaining samples (Fig. 3a).

Gammaproteobacteria and Betaproteobacteria is abundant in most samples, except for Gammaproteobacteria in $C x$. quinquefasciatus males $(<1 \%)$ and for Betaproteobacteria in $C x$. quinquefasciatus males and females, $A n$. stephensi and An. gambiae Kisumu females (Fig. 3a).

On the contrary, reproductive organs of the species of the An. gambiae complex harbour a dissimilar microbiota: the class of Bacilli is predominant, followed by Gammaproteobacteria, Actinobacteria, Betaproteobacteria and Fusobacteria. However, An. gambiae G3 represents an exception, where Gammaproteobacteria (85\%) in females and Flavobacteria (74\%) in males dominate the overall microbiota (Fig. 3a).

Despite a few exceptions, the analysis of taxa at genus level showed the persistence of a shared core of bacteria. An. gambiae G3 ovaries are mainly colonized by Serratia (78\%) followed by Escherichia-Shigella (0.5\%), Sphingomonas (0.2\%), Cupriavidus (0.2\%) and Elizabethkingia $(0.2 \%)$. The latter, on the contrary, represents the dominant taxon $(73 \%)$ in male organs, co-habiting with Burkholderia (18\%) and, in much lower amount, with Serratia (0.3\%). Similarly, in An. stephensi, Serratia predominantly inhabits male reproductive organs, while in 
Table 1 Bacterial diversity and richness in salivary glands, guts and reproductive organs of mosquito species

\begin{tabular}{|c|c|c|c|c|c|c|}
\hline Mosquitoes & Sex & Organ & $\begin{array}{l}\mathrm{N} . \\
\text { reads }\end{array}$ & $\begin{array}{l}\text { Observed } \\
\text { OTUs }\end{array}$ & Shannon & Simpson \\
\hline \multirow[t]{5}{*}{ An. gambiae G3 } & \multirow[t]{3}{*}{ q } & s.g. & 10,775 & 66 & 2038 & 0,752 \\
\hline & & guts & 18,555 & 222 & 2364 & 0,691 \\
\hline & & r.o. & 22,418 & 326 & 2719 & 0,739 \\
\hline & \multirow[t]{2}{*}{ 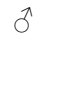 } & guts & 13,918 & 78 & 2013 & 0,746 \\
\hline & & r.o. & 13,918 & 78 & 1947 & 0,699 \\
\hline \multirow[t]{5}{*}{ An. coluzzii } & \multirow[t]{3}{*}{ q } & s.g. & 10,959 & 116 & 2229 & 0,766 \\
\hline & & guts & 17,160 & 157 & 1910 & 0,595 \\
\hline & & r.o. & 62,851 & 467 & 4930 & 0,979 \\
\hline & \multirow[t]{2}{*}{$\hat{0}$} & guts & 10,105 & 57 & 1561 & 0,650 \\
\hline & & r.o. & 62,732 & 448 & 4670 & 0,971 \\
\hline \multirow[t]{5}{*}{ An. quadriannulatus } & \multirow[t]{3}{*}{ q } & s.g. & 17,056 & 296 & 2903 & 0,797 \\
\hline & & guts & 22,176 & 236 & 3267 & 0,853 \\
\hline & & r.o. & 53,254 & 427 & 4813 & 0,974 \\
\hline & \multirow[t]{2}{*}{$\hat{\sigma}$} & guts & 18,000 & 137 & 1935 & 0,596 \\
\hline & & r.o. & 13,540 & 323 & 4373 & 0,954 \\
\hline \multirow{5}{*}{$\begin{array}{l}\text { An. gambiae } \\
\text { Kisumu }\end{array}$} & \multirow[t]{3}{*}{ q } & s.g. & 22,653 & 343 & 2772 & 0,744 \\
\hline & & guts & 15,551 & 239 & 3109 & 0,797 \\
\hline & & r.o. & 10,160 & 181 & 3354 & 0,910 \\
\hline & \multirow[t]{2}{*}{$\hat{0}$} & guts & 13,774 & 209 & 2693 & 0,843 \\
\hline & & r.o. & 59,029 & 472 & 4836 & 0,977 \\
\hline \multirow[t]{5}{*}{ An. arabiensis } & \multirow[t]{3}{*}{ q } & s.g. & 28,828 & 337 & 2865 & 0,831 \\
\hline & & guts & 20,354 & 192 & 2105 & 0,631 \\
\hline & & r.o. & 10,021 & 294 & 3605 & 0,907 \\
\hline & \multirow[t]{2}{*}{$\hat{\sigma}$} & guts & 26,666 & 465 & 3675 & 0,898 \\
\hline & & r.o. & 13,884 & 363 & 4567 & 0,968 \\
\hline \multirow[t]{5}{*}{ An. merus } & \multirow[t]{3}{*}{ q } & s.g. & 3553 & 175 & 3286 & 0,896 \\
\hline & & guts & 24,600 & 218 & 2530 & 0,754 \\
\hline & & r.o. & 2228 & 11 & 1211 & 0,619 \\
\hline & \multirow[t]{2}{*}{$\hat{0}$} & guts & 27,009 & 258 & 2941 & 0,853 \\
\hline & & r.o. & 5548 & 10 & 0,980 & 0,564 \\
\hline \multirow[t]{5}{*}{ An. stephensi } & \multirow[t]{3}{*}{ q } & s.g. & 23,533 & 196 & 2416 & 0,720 \\
\hline & & guts & 18,922 & 233 & 2103 & 0,605 \\
\hline & & r.o. & 15,628 & 302 & 3898 & 0,953 \\
\hline & \multirow[t]{2}{*}{$\hat{0}$} & gut & 16,619 & 157 & 1905 & 0,588 \\
\hline & & r.o. & 15,070 & 209 & 2647 & 0,782 \\
\hline \multirow[t]{5}{*}{ Ae. albopictus } & \multirow[t]{3}{*}{ o } & s.g. & 12,976 & 218 & 2395 & 0,687 \\
\hline & & guts & 17,025 & 387 & 3791 & 0,921 \\
\hline & & r.o. & 16,078 & 114 & 2607 & 0,866 \\
\hline & \multirow[t]{2}{*}{$\hat{0}$} & guts & 22,371 & 382 & 3759 & 0,913 \\
\hline & & r.o. & 8960 & 237 & 3372 & 0,894 \\
\hline Ae. aegypti & q & s.g. & 19,189 & 385 & 5019 & 0,975 \\
\hline & & guts & 20,603 & 427 & 4899 & 0,978 \\
\hline & & r.o. & 26,064 & 410 & 5025 & 0,973 \\
\hline
\end{tabular}

Table 1 Bacterial diversity and richness in salivary glands, guts and reproductive organs of mosquito species (Continued)

\begin{tabular}{llllllll}
\hline Mosquitoes & Sex & Organ & $\begin{array}{l}\text { N. } \\
\text { reads }\end{array}$ & $\begin{array}{l}\text { Observed } \\
\text { OTUs }\end{array}$ & Shannon & Simpson \\
& \multirow{2}{*}{ C. } & guts & 3167 & 184 & 3781 & 0,938 \\
& & r.o. & 5007 & 251 & 4382 & 0,965 \\
& o. quinquefasciatus & s.g. & 18,083 & 290 & 3304 & 0,885 \\
& & guts & 4268 & 146 & 2863 & 0,744 \\
& & r.o. & 2945 & 97 & 2777 & 0,840 \\
& \multirow{2}{*}{ o } & guts & 16,763 & 250 & 3744 & 0,888 \\
& & r.o. & 17,384 & 79 & 2835 & 0,874 \\
\hline
\end{tabular}

q: females; $\jmath^{2}$ : males; s.g.: salivary glands; r.o.: reproductive organs

ovaries it is exceeded by Asaia, whose presence in males is reduced. Finally, $C x$. quinquefasciatus offered a divergent scenario: male reproductive organs are mainly colonized by Phyllobacterium (91\%), although its contribution in ovaries microbiota was minimal (0.8\%) (Fig. 4a). Differently, the ovaries of $C x$. quinquefasciutus host

An. gambiae G3
An. coluzzii
An. quadriannulatus
An. gambiae Kisumu arabiensis
An. merus
An. stephensi
Ae. albopictus
Ae. aegypti
Cx. quinquefasciatus
Actinobacteria
Bacteroidetes
Cyanobacteria
Planctomycetes
nine mosquito species. All OTUs are represented, except the unassigned
I. males



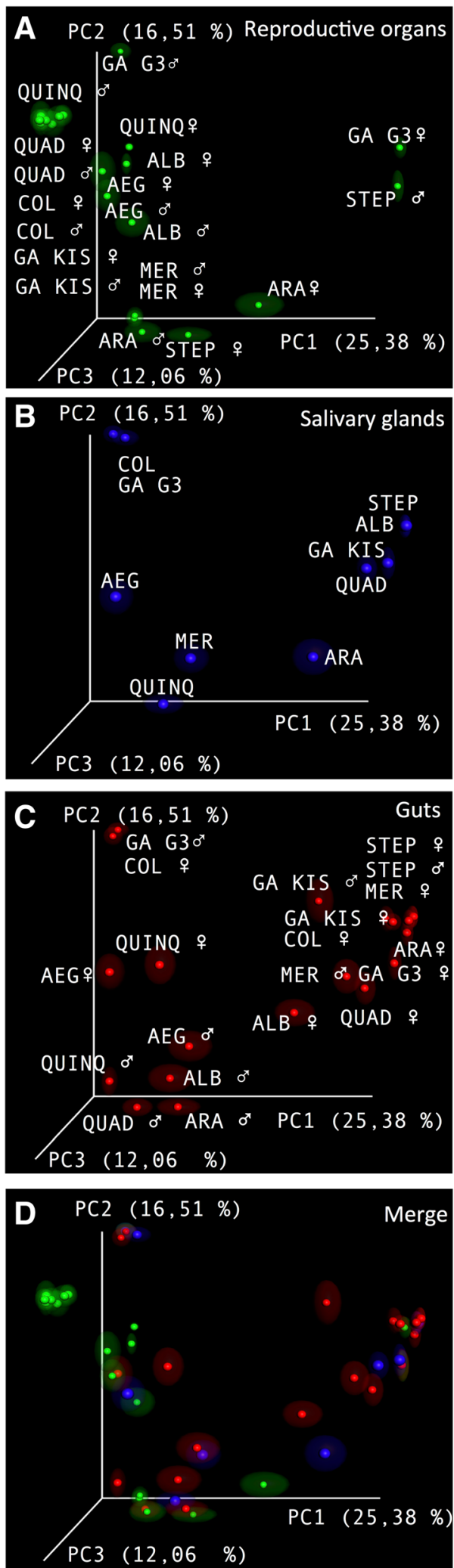

Fig. 2 Principal Coordinates Analysis (PCoA) plots of samples colored according to different organs. a Mosquitoes reproductive organs from females (i) and males $\left({ }^{\top}\right)$. b Salivary glands from female mosquito species. c Mosquito guts from females (o) and males $\left(\hat{O}^{\Uparrow}\right)$. $\mathbf{d}$ Merge figure from panel a, b and c. GA G3: An. gambiae G3; COL: An. coluzzii; QUAD: An. quadriannulatus; GA KIS: An. gambiae Kisumu; ARA: An. arabiensis; MER: An. merus; STEP: An. stephensi; ALB: Ae. albopictus; AEG: Ae. aegypti; QUINQ: CX. quinquefasciatus

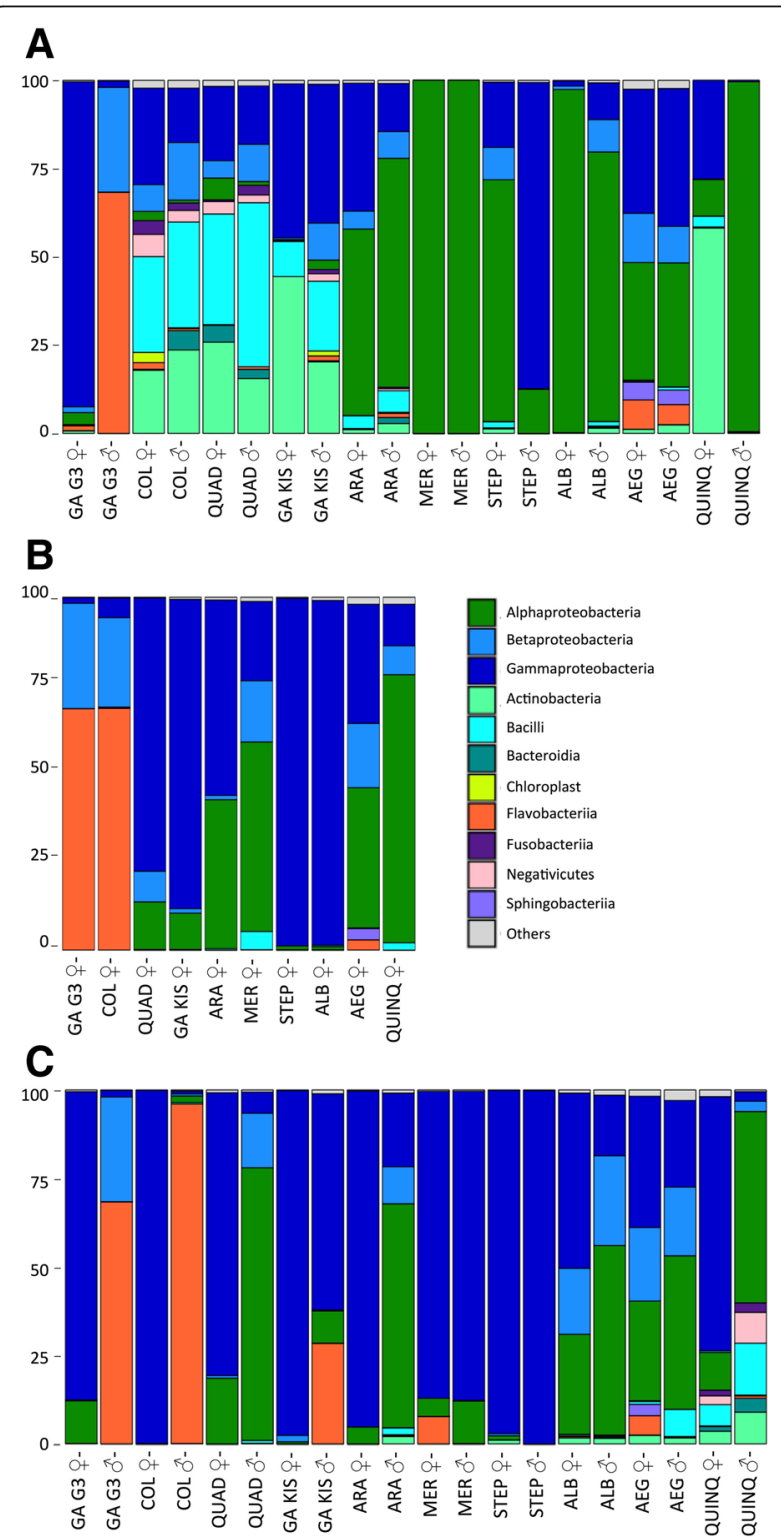

Fig. 3 Class level composition (\% of OTUs) in different organs of nine mosquito species. Only OTUs representing $>1 \%$ of the total reads are represented here. The class color code is given for (a), (b) and (c). GA G3: An. gambiae G3; COL: An. coluzzii; QUAD: An. quadriannulatus; GA KIS: An. gambiae Kisumu; ARA: An. arabiensis; MER: An. merus; STEP: An. stephensi; ALB: Ae. albopictus; AEG: Ae. aegypti; QUINQ: CX. quinquefasciatus; : : females; ${ }^{\top}$ : males 


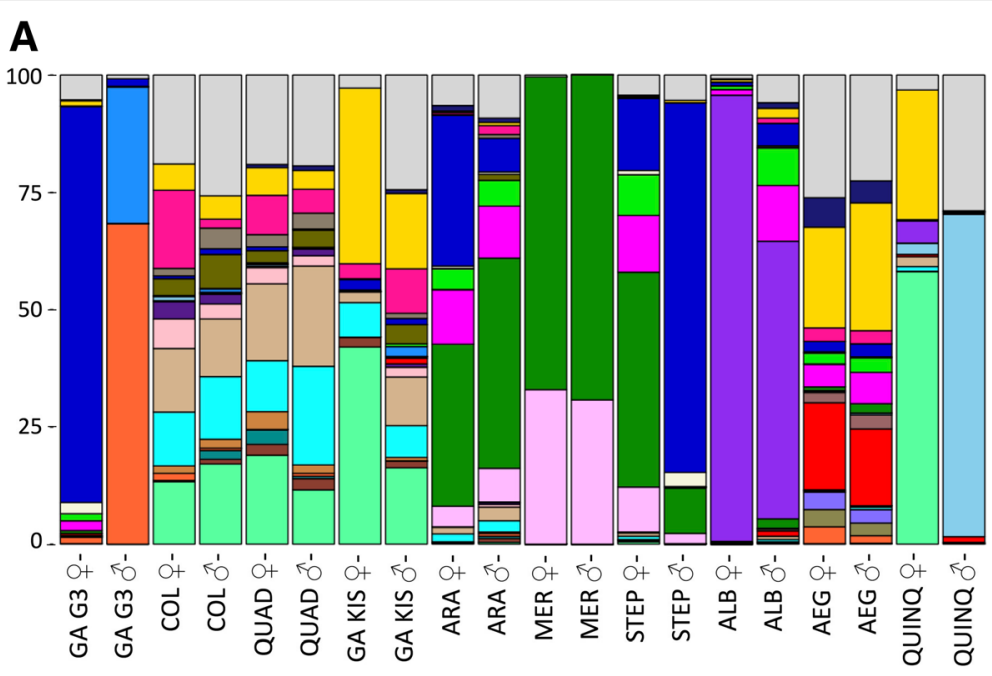

B
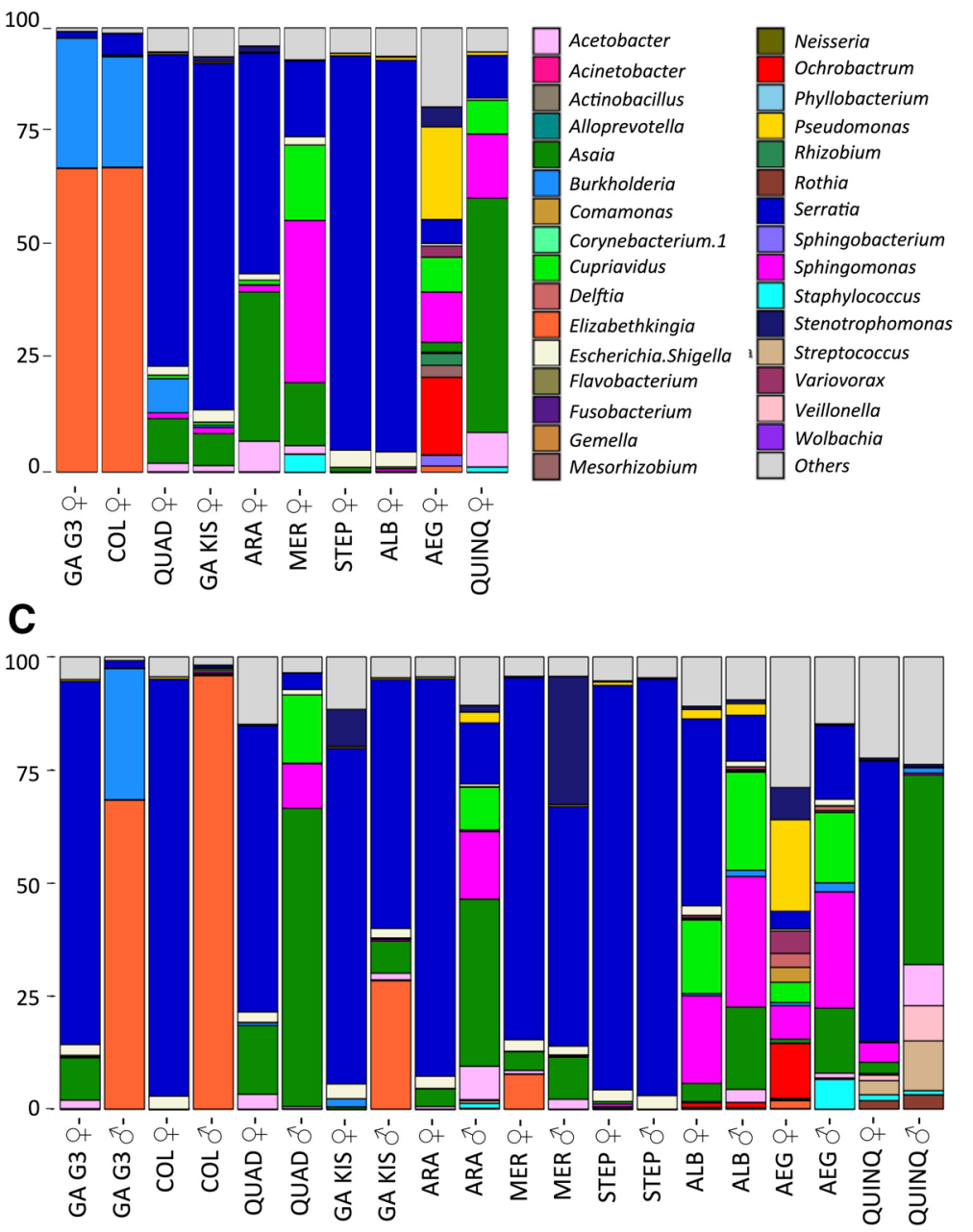

Fig. 4 Genus level composition (\% of OTUs) in different organs of nine mosquito species. Only OTUs representing > 1\% of the total reads are represented here. The genus color code is given for (a), (b) and (c). GA G3: An. gambiae G3; COL: An. coluzzii; QUAD: An. quadriannulatus; GA KIS: An. gambiae Kisumu; ARA: An. arabiensis; MER: An. merus; STEP: An. stephensi; ALB: Ae. albopictus; AEG: Ae. aegypti; QUINQ: CX. quinquefasciatus; O+: females; $\hat{O}^{2}$ : males 
Serratia, and several genera of the families Rhodobacteriaceae and Rhizobiaceae (Additional file 4: Figure S3). As expected, Wolbachia colonization in Ae. albopictus shows a noticeable predominance in ovaries (94\%); in male reproductive organs, it coexists with Sphingomonas, Cupriavidus and Serratia.

Finally, the reproductive organs of Ae. aegypti show an interestingly high richness of taxa: Ochrobactrum, Pseudomonas, Sphingomonas, Stenothropomonas, Acinetobacter and Elizabethkinghia are the most abundant genera detected in female and male individuals (Fig. 4a).

\section{Microbiota composition of salivary glands}

Female salivary glands are also collected and analysed. Gammaproteobacteria represents the most prevalent class in anophelines (An. quadriannulatus, An. gambiae Kisumu, An. arabiensis, and An. stephensi) and in Ae. albopictus, and, although less abundant, in An. merus, in Ae. aegypti and in Cx. quinquefasciatus. Alphaproteobacteria, followed by Betaproteobacteria, constitutes the remaining portion (Fig. 3b).

Serratia is present in each sample of salivary glands, and together with Escherichia-Shigella, Pantoea, Acetobacter, Sphingomonas, Burkholderia and Cupriavidus is part of a shared core of taxa. An. quadriannulatus, An. gambiae Kisumu, An. arabiensis are prevalently colonized by Serratia and Asaia. An. merus hosts Sphingomonas (34\%), Asaia (14\%) and in lower proportion Serratia and Cupriavidus. A completely divergent pattern is visible in An. gambiae G3 and An. coluzzii mosquitoes, where $69 \%$ of OTUs are addressed to the Elizabethkingia sp. genus, whose abundance reaches almost undetectable levels in the salivary glands of other species (apart from $1.4 \%$ in Ae. aegypti). In addition, salivary glands of $A n$. gambiae G3 and An. coluzzii are inhabited by Betaproteobacteria (genus Burkholderia, > $25 \%)$, whose colonization is found in lower amount (7.6\%) in An. quadriannulatus samples.

Ae. albopictus is mainly colonized by Serratia (88\%) like An. stephensi. Similarly to other organs, a conserved core of communities is found in salivary glands, consisting of Asaia, Pseudomonas, Sphingomonas, Cupriavidus, Serratia and Escherichia-Shigella. Moreover, other taxa are identified, including Acetobacter and Ochrobactrum (Alphaproteobacteria) and Elizabethkingia (Bacteroidetes) (Fig. 4b).

\section{Microbiota composition of guts}

The most diffused bacteria classes in anopheline is Gammaproteobacteria, mainly represented by Serratia, apart from An. gambiae G3 and An. coluzzii males, where Flavobacteria (represented by Elizabethkingia) reaches $78 \%$ and $95 \%$ of OTUs, respectively (Fig. 3c, Fig. 4c). Aedes microbiota is characterized by a higher diversity in guts, showing Alpha, Beta and Gammaproteobacteria equally colonizing the intestinal tissues (Fig. 3c). Detected genera are also Sphingomonas, Asaia, Cupriavidus, Escherichia-Shigella, Pseudomonas and Serratia (Fig. 4c). Finally, Cx. quinquefasciatus shows a unique composition, disclosing a variety of genera; however, Alphaproteobacteria (Asaia) are dominant in females, while Gammaproteobacteria (Yersinia) largely colonize male guts (Fig. 3c, Fig. 4c).

\section{Discussion}

Through a $16 \mathrm{~S}$ rRNA gene sequencing-based approach, a description of microbiota diversity hosted in salivary glands, reproductive tracts and guts of several mosquito species is provided. High richness and diversity of microbes associated with laboratory reared individuals was detected, suggesting the persistence of a well-established and conserved core of bacteria. The evidence of a shared microbiota core is consistent with other studies reporting similar data and identifying a large conserved group of bacteria colonizing different mosquito species and their tissues, also in standardized rearing environments. [19, 23, 24].

We are aware that bacterial DNA contamination in extraction kits and laboratory reagents can significantly affect the results of microbiota studies, particularly when samples contain low microbial biomass and when highly sensitive techniques are used. Previous studies described the list of contaminants detected in sequenced negative blank controls derived from reagents contamination, users and extraction kits [25]. The proposed list of contaminants has been compared to the obtained data. Only a few candidates, like Mesorhizobium, Phyllobacterium, Rhizobium, Comamonas, Delftia, Variovorax, Escherichia-Shigella were detected at very low percentages in our samples. It has been also reported that Corynebacterium, Propionibacterium and Streptococcus are common human skin-associated organisms. Only Corynebacterium and Streptococcus were detected in very low percentage $(<1 \%)$ in some samples. Overall, the incidence of bacteria contaminations in our data appears minimal; moreover, the taxa described above have been previously presented in metagenomics studies of insects, and for this reason they have been included in the data analysis.

Previous studies proved that commensal bacteria are mainly acquired from the environment or transmitted directly between hosts to their offspring. Often, these contributions are correlated, meaning that bacteria with complete transmission among hosts will evolve specialization for a particular niche [26-28]. Although bacterial composition is selected by standardized diet and rearing conditions, differences have been disclosed and data are able to provide crucial insights for a robust characterization and comparison between species. Additionally, despite belonging to the same individual, 
tissues specifically harboured diverse bacteria, whose distribution reflects tissue-bacteria adaptations. Within the same individual, microbiota of reproductive organs was more diverse than that of the gut and salivary glands. Salivary glands, mostly in anophelines, showed higher diversity indices when compared to the guts, similarly to what reported in An. culicifacies [20]. A leading example of this characteristic adaptation is Elizabethkingia: it largely colonizes guts and reproductive organs of An. gambiae G3 males, while in An. coluzzii, it is prevalent in guts of males and salivary glands of females. Its association with guts of An. gambiae was already reported in mosquitoes reared in standard insectary conditions, suggesting the establishment of an own thriving niche, and possibly explained with the ability of Bacteroidetes to degrade sugar [29]. Similarly, Serratia, although presenting variable abundances in each sample, is found to be dominant in the gut of female anophelines, being consistent with recently reported data [19, 30]. For instance, in An. stephensi, Serratia represents more than $90 \%$ of detected taxa in all organs, except for female ovaries where its abundance is reduced.

Notably, Asaia occurrence is in line with its role among symbiotic bacterial communities: it rarely showed, in fact, dominant behaviour, like Serratia or Elizabethkingia, but its persistence is found in guts and salivary glands of all mosquito genera and its relevant occurrence in reproductive organs of males and females of An. stephensi and An. arabiensis provides evidences of its widespread colonization ability.

Bacteria such as Asaia, Pantoea, Pseudomonas, Entrobacter, Serratia are gaining momentum as promising candidates for paratransgenic modifications for vector control strategies. Our findings confirm their abundance in key tissues of various mosquito species, corroborating and widening their role as broad-spectrum tools against mosquito borne diseases. Cupriavidus and Ochrobactrum could be also included in this bacterial arsenal: their association with mosquitoes was never reported before, but their recent engineering for biomedical purposes could be also translated in the field of mosquito control [31, 32]. In addition, the presence of some bacteria as Serratia and Wolbachia in different mosquito species are well-known to provide protective effects from pathogens infections, as reported in several studies [33, 34].

In any case, before any field application of bacterial strains, a detailed characterization and analysis of the potential risks for human and animal health is an imperative.

\section{Conclusions}

Our study describes the composition of microbial communities harboured in different tissues of nine mosquito species, transmitting devastating human diseases. The results highlight the importance of a comprehensive understanding of organisms, intended as holobionts, and thus composed by innumerable interacting communities, whose impact is increasingly apparent. The identification of interesting inter- and intra-species differences, coexisting with a shared core microbiota, suggest a singular adaptation and a tissue-specific tropism. The contextual findings of a deep degree of divergences between genera, underlines microbiota specificity and its adaptation to the host. Moving forward, our data lay the basis for the design of effective vector control strategies, relying on the use of mosquito-associated bacteria.

\section{Methods}

\section{Mosquito strains}

The following laboratory strains were used:

An. gambiae G3 (MR4, MRA-112) established in Perugia insectary in 2013; An. gambiae Kisumu (MR4, MRA-762) established in Perugia insectary in 2013; $A n$. coluzzii (MR4, MRA-860) established in Perugia insectary in 2013 (it is worth to mention that we analysed two strains of An. gambiae, due to the high polymorphism of G3, comparing whether the genetic background could influence the microbiota composition); An. arabiensis (MR4, MRA-339) established in Perugia insectary in 2013; An. merus (MR4, MRA1156) established in Perugia insectary in 2013; An. quadriannulatus (MR4, MRA-761) established in Perugia insectary in 2013; An. stephensi (SD-500) established in Perugia insectary in 2011; Ae. albopictus (MR4, MRA-804) established in Perugia insectary in 2013; Ae. aegypti (New Orleans, LA 2011) established in Perugia insectary in 2013; Cx. quinquefasciatus (collected Hawaii U.S.A., 2008 and provided by Rutgers University) established in Camerino insectary in 2011.

\section{Mosquito rearing}

Analyzed mosquitoes were all cyclic laboratory-strain colonies. Mosquitoes were reared with a $12 \mathrm{~h}$ day/night cycle at $27{ }^{\circ} \mathrm{C}$ and relative humidity of $70 \%$. Anopheles, Aedes and Culex larvae were reared in deionized water with $0.3 \mathrm{~g} /$ liter of artificial sea salts and fed daily with a diet composed by a slurry of 2:2:1 bovine liver powder, tuna meal and Vanderzant vitamin mix [35]. Adults were fed ab libitum on $10 \%$ sucrose.

\section{Tissues collection and DNA extraction}

Prior dissecting, mosquitoes were surface sterilized in $70 \%$ ethanol for $5 \mathrm{~min}$ and rinsed twice in sterile PBS. Foreguts and midguts, salivary glands lobes and reproductive organs, consisting on testes and male accessory glands (MAGs) for males, and ovaries for females, were dissected with special care to reduce contaminations: needles and dissecting slides were sterilized and treated with $70 \%$ ethanol between each sample, while storing vials were UV treated before use. Pooled guts (10 whole guts), 
reproductive organs (10 sets) and salivary glands (20 pairs) were homogenized with sterile $0.5-\mathrm{mm}$ wide glass beads (Bertin) for 30s at $6800 \mathrm{rpm}$ in the automatic tissue homogenizer (Precellys 24, Bertin). Genomic DNA was extracted using a JetFlex Genomic DNA Purification kit (Genomed) according to the manufacturer's instructions.

\section{Amplicon and library preparation}

Bacterial 16S V4 regions rDNA were amplified using bacteria/archaeal degenerate primers 515F/806R [36]. Amplification and library preparation were performed as reported previously [37].

\section{Bioinformatic analysis}

Quality control of raw data was done using FastQC, then adapters sequences and low quality scores $(<20)$ were trimmed by Trimmomatic software package [38]. Cleaned sequenced paired-end reads were merged to reconstruct original full-length $16 \mathrm{~S}$ amplicons with PEAR software [39]. All amplicons with sequence similarity higher than 97\% were chosen as input for making the taxonomy annotation and building the OTU table by following the Open Reference approach. The obtained sequences were searched for matching in the SILVA taxonomy database (v123) using similarity-searching algorithm [40]. The microbial communities of each sample were built from the taxonomy assignments. The alpha-diversity (of each sample) was investigated by means of three different indexes: Shannon and Simpson observed OTUs, in order to quantify the number and distribution of taxa in each sample [41-43]. The dissimilarity analysis between samples measuring population composition (beta-diversity) has been evaluated using QIIME weighted Unifrac distances between samples at a sub-sampling depth of 100 sequences per sample. Thus, jackknifed principal coordinates have been calculated on rarefied results to compress dimensionality into two- and three-dimensional principal coordinate analysis plots. The rarefied results have been used also for computing alpha diversity using observed species, Shannon and phylogenetic diversity (PD) metrics. Data analysis was done using Qiime and the R-software (http://qiime.org, http://www.r-project.org/).

\section{Additional files}

Additional file 1: Figure S1. Alpha diversity. Box plots of bacterial species richness associated with the nine mosquito species. The box plots indicate median (middle line), upper and lower quartiles (box top and bottom), minimum and maximum (whiskers). (JPG $3163 \mathrm{~kb}$ )

Additional file 2: Figure S2. Rarefaction curves. Rarefaction curves calculated for each sample based on the OTU computations, reflect different diversities in different samples and help to estimate whether bacterial communities were sampled properly, i.e. enough sequence reads per sample where collected. Rarefaction curves are expected to reach a plateau if sampling has been exhaustive. O+: females; $\hat{0}$ : males. (JPG $1442 \mathrm{~kb})$

Additional file 3: Table S1. Percentage of phyla OTUs in the reproductive organs (A) salivary glands (B) and guts (C). (DOCX $47 \mathrm{~kb}$ )

Additional file 4: Figure S3. Family level composition (\% of OTUs) in different organs of nine mosquito species. Only OTUs representing $>1 \%$ of the total reads are represented here. The family color code is given for (A), (B) and (C). GA G3: An. gambiae G3; COL: An. coluzzii; QUAD: An. quadriannulatus; GA KIS: An. gambiae Kisumu; ARA: An. arabiensis; MER: An. merus; STEP: An. stephensi; ALB: Ae. albopictus; AEG: Ae. aegypti; QUINQ: CX. quinquefasciatus; 9 : females; $\widehat{0}$ : males. (JPG $3132 \mathrm{~kb}$ )

\section{Abbreviations}

BLAST: basic local alignment search tool; MAGs: male accessory glands; MR4: The Malaria Research and Reference Reagent Resource Centre; NCBI: National Centre for Biotechnology Information; OTU: operational taxonomic unit; PBS: phosphate buffer saline solution; PCoA: principal coordinate analysis; PD: phylogenetic diversity; PEAR: paired-end read merger; QIIME: quantitative insights into microbial ecology; rRNA: ribosomal RNA; SC: Symbiotic Control

\section{Funding}

This work was supported by the Italian Ministry for Education and Research (MIUR), awards numbers Prin 2012-2012T85B3R and PRIN 2015-JXC3JF, both to GF, and by the University of Camerino, award number FAR2015 to GF. Funding bodies had no role in the design of the study and collection, analysis, and interpretation of data and in writing the manuscript.

\section{Availability of data and materials}

Raw metagenomic reads are deposited in the NCBI, BioProject ID: PRJNA416453. Web address https://www.ncbi.nlm.nih.gov/bioproject/ ?term=PRJNA416453

\section{Authors' contributions}

GF, MVM, CD, and RS conceived and designed the experiments. GF and RS supervised the project and gave conceptual advice. MVM, CD, AA, MT, PR, $M V, J B, A S, I R, R S, R C$ and GF participated in the sample collection. MVM, $C D, A C$, and $A A$ performed the DNA extractions. MVM, CD, AA, EN, and RS recollected all the metadata. EN carried out the bioinformatics analysis. GF and RS drafted the manuscript. GF, MVM, CD, and RS edited the manuscript. All authors read and approved the final manuscript.

Ethics approval and consent to participate

Research carried out on invertebrate such as mosquitoes do not require a specific permit according to the directive 2010/63/EUof the European Parliament and of the Council on the protection of animals used for scientific purposes.

Consent for publication

Not applicable.

Competing interests

The authors declare that they have no competing interest.

\section{Publisher's Note}

Springer Nature remains neutral with regard to jurisdictional claims in published maps and institutional affiliations.

\section{Author details}

${ }^{1}$ School of Biosciences and Medical Veterinary, University of Camerino, Via Gentile III da Varano, 62032 Camerino, MC, Italy. ${ }^{2}$ Department of Experimental Medicine, Functional Genomics Center, University of Perugia, Via Lucio Severi 1, 06132 Perugia, Italy. ${ }^{3}$ Present Address: Centre for Virus Research, Level 3 Henry Wellcome Building, 464 Bearsden Road, Glasgow, UK. 
Received: 15 February 2018 Accepted: 26 September 2018 Published online: 04 October 2018

\section{References}

1. Basset $Y$, Cizek L, Cuénoud P, Didham RK, Guilhaumon F, Missa O, et al. Arthropod diversity in a tropical forest. Science. 2012;338:1481-4.

2. Dillon RJ, Dillon VM. The gut bacteria of insects: nonpathogenic interactions. Annu Rev Entomol. 2004;49:71-92.

3. Latorre A, Manzano-Marín A. Dissecting genome reduction and trait loss in insect endosymbionts. Ann N Y Acad Sci. 2017;1389:52-75.

4. Sharon G, Segal D, Ringo JM, Hefetz A, Zilber-Rosenberg I, Rosenberg E. Commensal bacteria play a role in mating preference of Drosophila melanogaster. Proc Natl Acad Sci U S A. 2010;107:20051-6.

5. Goodacre SL, Martin OY. Modification of insect and arachnid Behaviours by vertically transmitted Endosymbionts: infections as drivers of Behavioural change and evolutionary novelty. Insects. 2012;3:246-61.

6. Leonardo TE, Mondor EB. Symbiont modifies host life-history traits that affect gene flow. Proc Biol Sci. 2006;273:1079-84

7. Coon KL, Vogel KJ, Brown MR, Strand MR. Mosquitoes rely on their gut microbiota for development. Mol Ecol. 2014;23:2727-39.

8. Kikuchi Y, Hayatsu M, Hosokawa T, Nagayama A, Tago K, Fukatsu T. Symbiont-mediated insecticide resistance. Proc Natl Acad Sci U S A. 2012; 109:8618-22.

9. Patterson J, Sammon M, Garg M. Dengue, Zika and Chikungunya: emerging Arboviruses in the New World. West J Emerg Med. 2016;17:671-9.

10. Sudeep AB, Mandar P, Ghodke YK, George RP, Gokhale MD. Vector competence of two Indian populations of Culex quinquefasciatus (Diptera: Culicidae) mosquitoes to three West Nile virus strains. J Vector Borne Dis. 2015;52:185-92

11. Clements AN. The biology of mosquitoes: sensory reception and behaviour. Wallingford: CABI Publishing; 1999.

12. Dong Y, Manfredini F, Dimopoulos G. Implication of the mosquito midgut microbiota in the defense against malaria parasites. PLoS Pathog. 2009;5: e1000423.

13. Ryu JH, Kim SH, Lee HY, Bai JY, Nam YD, Bae JW, et al. Innate immune homeostasis by the homeobox gene caudal and commensal-gut mutualism in Drosophila. Science. 2008:319:777-82.

14. Gaio Ade O, Gusmão DS, Santos AV, Berbert-Molina MA, Pimenta PF, Lemos FJ. Contribution of midgut bacteria to blood digestion and egg production in Aedes aegypti (diptera: culicidae) (L.). Parasit Vectors. 2011;4:105.

15. Ramirez JL, Short SM, Bahia AC, Saraiva RG, Dong Y, Kang $S$, et al. Chromobacterium Csp_P reduces malaria and dengue infection in vector mosquitoes and has entomopathogenic and in vitro anti-pathogen activities. PLoS Pathog. 2014;e1004398:10.

16. Dodson BL, Hughes $\mathrm{GL}$, Paul O, Matacchiero AC, Kramer LD, Rasgon JL. Wolbachia enhances West Nile virus (WNV) infection in the mosquito Culex tarsalis. PLoS Negl Trop Dis. 2014;8:e2965.

17. Apte-Deshpande A, Paingankar M, Gokhale MD, Deobagkar DN. Serratia odorifera a midgut inhabitant of Aedes aegypti mosquito enhances its susceptibility to dengue-2 virus. PLoS One. 2012;7:e40401.

18. Dharne M, Patole M, Shouche YS. Microbiology of the insect gut: tales from mosquitoes and bees. J Biosci. 2006:31:293-5.

19. Tchioffo MT, Boissière A, Abate L, Nsango SE, Bayibéki AN, Awono-Ambéné $\mathrm{PH}$, et al. Dynamics of bacterial community composition in the malaria Mosquito's epithelia. Front Microbiol. 2016;6:1500.

20. Sharma P, Sharma S, Maurya RK, Das De T, Thomas T, Lata S, et al. Salivary glands harbor more diverse microbial communities than gut in Anopheles culicifacies. Parasit Vectors. 2014:7:235.

21. Wang S, Jacobs-Lorena M. Genetic approaches to interfere with malaria transmission by vector mosquitoes. Trends Biotechnol. 2013;31(3):185-93.

22. Bokulich NA, Subramanian S, Faith JJ, Gevers D, Gordon Jl, Knight R, et al. Quality-filtering vastly improves diversity estimates from Illumina amplicon sequencing. Nat Methods. 2013:10:57-9.

23. Dickson LB, Ghozlane A, Volant S, Bouchier C, Ma L, Vega-Rúa A, et al. Diverse laboratory colonies of Aedes aegypti harbor the same adult midgut bacterial microbiome. Parasit Vectors. 2018;11(1):207.

24. Segata N, Baldini F, Pompon J, Garrett WS, Truong DT, Dabiré RK, et al. The reproductive tracts of two malaria vectors are populated by a core microbiome and by gender- and swarm-enriched microbial biomarkers. Sci Rep. 2016;6:24207.
25. Salter SJ, Cox MJ, Turek EM, Calus ST, Cookson WO, Moffatt MF, et al. Reagent and laboratory contamination can critically impact sequence-based microbiome analyses. BMC Biol. 2014;12:87.

26. Kikuchi Y, Hosokawa T, Fukatsu T. Insect-microbe mutualism without vertical transmission: a stinkbug acquires a beneficial gut symbiont from the environment every generation. Appl Environ Microbiol. 2007;73:4308-16.

27. Moran NA, McCutcheon JP, Nakabachi A. Genomics and evolution of heritable bacterial symbionts. Annu Rev Genet. 2008;42:165-90.

28. Gimonneau G, Tchioffo MT, Abate L, Boissière A, Awono-Ambéné PH, Nsango SE, et al. Composition of Anopheles coluzzii and Anopheles gambiae microbiota from larval to adult stages. Infect Genet Evol. 2014:28:715-24.

29. Boissière A, Tchioffo MT, Bachar D, Abate L, Marie A, Nsango SE, et al. Midgut microbiota of the malaria mosquito vector Anopheles gambiae and interactions with Plasmodium falciparum infection. PLoS Pathog. 2012; e1002742:8.

30. Wang S, Dos-Santos ALA, Huang W, Liu KC, Oshaghi MA, Wei G, et al. Driving mosquito refractoriness to Plasmodium falciparum with engineered symbiotic bacteria. Science. 2017;357:1399-402.

31. Guo KH, Chen PH, Lin C, Chen CF, Lee IR, Yeh YC. Determination of gold ions in human urine using genetically engineered microorganisms on a paper device. ACS Sens. 2018;3(4):744-8.

32. He Y, Vemulapalli R, Schurig GG. Recombinant Ochrobactrum anthropi expressing Brucella abortus $\mathrm{Cu}, \mathrm{Zn}$ superoxide dismutase protects mice against $B$. abortus infection only after switching of immune responses to Th1 type. Infect Immun. 2002;70(5):2535-43.

33. Bahia AC, Dong Y, Blumberg BJ, Mlambo G, Tripathi A, BenMarzouk-Hidalgo OJ, et al. Exploring Anopheles gut bacteria for Plasmodium blocking activity. Environ Microbiol. 2014;16(9):2980-94.

34. Ant HT, Herd CH, Geoghegan V, Hoffmann AA, Sinkins SP. The Wolbachia strain wAu provides highly efficient virus transmission blocking in Aedes aegypti. PLoS Pathog. 2018;14(1):e1006815.

35. Damiens D, Benedict MQ, Wille M, Gilles JR. An inexpensive and effective larval diet for Anopheles arabiensis (Diptera: Culicidae): eat like a horse, a bird, or a fish? J Med Entomol. 2012;49:1001-11.

36. Caporaso JG, Lauber CL, Walters WA, Berg-Lyons D, Huntley J, Fierer N, et al. Ultra-high-throughput microbial community analysis on the Illumina HiSeq and MiSeq platforms. ISME J. 2012:6:1621-4.

37. Mancini MV, Spaccapelo R, Damiani C, Accoti A, Tallarita M, Petraglia E, et al. Paratransgenesis to control malaria vectors: a semi-field pilot study. Parasit Vectors. 2016:9:140.

38. Bolger AM, Lohse M, Usadel B. Trimmomatic: a flexible trimmer for Illumina sequence data. Bioinformatics. 2014:30:2114-20.

39. Zhang J, Kobert K, Flouri T, Stamatakis A. PEAR: a fast and accurate Illumina paired-end reAd mergeR. Bioinformatics. 2014;30:614-20.

40. Quast C, Pruesse E, Yilmaz P, Gerken J, Schweer T, Yarza P, et al. The SILVA ribosomal RNA gene database project: improved data processing and webbased tools. Nucleic Acids Res. 2013;41:D590-6.

41. Simpson EH. Measurement of diversity. Nature. 1949;163:688.

42. Shannon CE. A mathematical theory of communication. BSTJ. 1948;27:3.

43. Fisher RA, Steven Corbet A, Williams CB. The relation between the number of species and the number of individuals in a random sample of an animal population. J Anim Ecol. 1943;2:42-58.

Ready to submit your research? Choose BMC and benefit from:

- fast, convenient online submission

- thorough peer review by experienced researchers in your field

- rapid publication on acceptance

- support for research data, including large and complex data types

- gold Open Access which fosters wider collaboration and increased citations

- maximum visibility for your research: over $100 \mathrm{M}$ website views per year

At BMC, research is always in progress.

Learn more biomedcentral.com/submission 